\title{
Propuesta de mejora energética para una planta de producción de clinker
}

\author{
Energy improvement proposal for a \\ clinker production plant
}

\author{
Leonardo Rodriguez Urrego \\ Universidad EAN, Bogotá - Colombia \\ Jeffrey León Pulido \\ Universidad EAN, Bogotá - Colombia \\ Andrés Cruz Pérez \\ Universidad EAN, Bogotá - Colombia \\ Hansen Murcia \\ Universidad Nacional, Bogotá - \\ Colombia \\ Mateo Gómez Velásquez \\ Universidad de America, Bogotá - \\ Colombia \\ Daniella Rodríguez Urrego \\ Universidad Piloto de Colombia, \\ Bogotá - Colombia
}

Nadson Lima

Autor de correspondencia

Universidade Estadual de

Campinas, Av. Albert Einstein

- 500, Campinas - Brasil.

Correo electronico: 1rodriguezu@ universidadean.edu.co

Información del artículo: recibido: 25 de Abril de 2017, aceptado: 12 de Julio de 2017 https://10.17081/invinno.5.2.2754 


\section{Resumen}

El siguiente trabajo presenta un análisis de la eficiencia energética en la industria cementera y un ejemplo real en donde se proponen mejoras para disminuir considerablemente el consumo energético. El aumento de la eficiencia disminuye considerablemente el costo de la energía eléctrica, además de las emisiones de CO2 ayudando así al mantenimiento y la preservación del medio ambiente. El caso de estudio fue aplicado en la planta de producción de cemento de san Vicente del Raspeig operada por CEMEX en España. En este caso se analizó una sección de la planta de producción, donde se realiza la molienda y el ensacado de cemento. Se encontró que, al implementar las mejoras propuestas, el costo de producción disminuye y el punto de equilibrio con respecto a la implementación de estos nuevos equipos no era mayor a 5 años lo cual hace que estas mejoras sean factibles.

\section{Abstract}

This paper presents an analysis of the energy efficiency in the cement industry and is a real example of where improvements are proposed in order to significantly reduce the use of energy. On the other hand, it was observed an increasing efficiency; the monetary cost of electric power is considerably reduced as well as the CO2 emissions, thus helping the maintenance and preservation of the environment. The case study was developed at a cement factory in San Vicente del Raspeig operated by CEMEX in Spain. Here a section of the production plant, in which grinding and cement packing is carried, was analyzed. It was found that when implementing the proposed improvements, the cost of production decreased and the point of equilibrium with respect to the implementation of the new equipment was not more than five years, making with these improvements a feasible project.

\section{Palabras \\ Claves: \\ Eficiencia energética; emisión de CO2, molienda; clinker.}

\section{Keywords:}

Energy efficiency, CO2 emission, grinding, clinker 


\section{Introducción}

Uno de los grandes retos de la ingeniería moderna es mejorar la eficiencia energética de los procesos productivos. El Banco Mundial propuso diferentes metas al respecto, llamadas los retos del milenio. Entre ellos destacan la disminución de las emisiones de $\mathrm{CO} 2$ y la generación de procesos sostenibles y eficientes para evitar la destrucción del medio ambiente a las nuevas generaciones [1]

Dado lo anterior, se han empezado a implementar nuevas alternativas en diferentes industrias, de modo que sus diferentes procesos productivos sigan operando pero de manera sostenible. Algunos casos de alternativas en diferentes industrias son: La producción de energía eléctrica utilizando hidrógeno como vector energético [2-4]. La implementación de nuevas tecnologías para la absorción de CO2 [5]. La implementación de energías alternativas como la fotovoltaica [6] y así mismo la implementación de nuevas tecnologías para la mejora de la eficiencia energética en las diferentes industrias [7-9].. Y, justamente, este trabajo analiza la producción de cemento, material clave hoy en día en diversas aplicaciones de orden mundial como: construcción, diversos pavimentos, fabricación de baldosas etc.,

En efecto, una de las industrias más importantes en la actualidad es la cementera. Esta industria consume alrededor del 12-15\% del uso energético industrial [10], lo que la constituye en una de las más grandes consumidoras energéticas. Es por esto que se han empezado a generar nuevas tecnologías con el fin de aumentar la eficiencia energética en la producción de clinker, tanto a nivel técnico en cuanto a la maquinaria instalada, como a nivel de sus procesos logísticos de cadena de suministro [11]. También se busca en este campo la eficiencia en procesos de mantenimiento predictivo, proactivo y de la condición en sistemas complejos $[12,13]$. Sin embargo, aún existen diferentes plantas en el mundo que tienen más de 30 años y siguen funcionando con tecnología de vanguardia. Debido a esto, es necesario realizar análisis energéticos y observar la viabilidad para implementar mejoras que hagan más eficiente el proceso desde el punto de vista energético y económico con el fin de aumentar el beneficio. 
En este trabajo se presentan concretamente la evaluación y las medidas planteadas para la implementación de un proceso de mejora energética respecto a la eficiencia y a la implementación de técnicas alternativas que permitan optimizar los recursos de energía y gas en algunos de los procesos más significativos de esta industria cementera. Este proceso fue llevado a cabo durante 6 meses por expertos en el área de procesos, energías alternativas y eficiencia energética, que fueron asistidos, a su vez, por una compañía consultora española. Los procesos analizados fueron los que acarrean mayor consumo energético, como la molienda, los exhaustores, los compresores y la iluminación. Los resultados obtenidos revelan un análisis económico exhaustivo de la TIR respecto a los factores de costo energético actual, costo de implementación, costo energético después de la implementación, y mantenimiento.

\section{Descripción de la Planta}

Como se mencionó al inicio del documento, la instalación auditada es la planta que la empresa CEMEX tiene en San Vicent del Raspeig (Alicante, España), donde se realiza el proceso productivo para la obtención del cemento Portland gris principalmente.

La planta se construyó en el año 1975. No obstante, algunas de sus instalaciones se han renovado con el tiempo.

Las entradas de materias primas a la cementera son:

Combustibles: Coque de petróleo, Enerfuel (combustible derivado de residuos), neumáticos troceados, harinas cárnicas y lodos de depuradora. Además, en los encendidos del horno se usa fuel y gasóleo.

Minerales: Caliza y marga, que provienen de una cantera cercana; escorias de horno extraídas del mismo, y arena de rodeno, yeso y sulfato ferroso comprados a otras empresas. Como resultado del proceso se obtienen: Cemento Portland gris, de los tipos: I 52,5 R; I 52,5 N/SR; II/ B-LL 32,5 N; II/B-M(S-LL) 42,5 R., clínker y gases calientes. 


\section{Procedimiento}

Con el fin de proponer mejoras para la eficiencia energética, primero fue necesario realizar el análisis energético actual de la planta de producción. Para este análisis, se tomó en cuenta la cantidad de energía consumida por la sección de la planta a utilizar en los diferentes meses del año, dividiéndola en dos grupos principales: días laborales y días festivos; estos a su vez se dividieron en horas, puesto que en esta planta el precio de la energía eléctrica varía según la hora en la cual se utiliza. Para concretar un análisis general, se realizó un promedio de consumo anual, obteniéndose lo presentado en la Gráfica 1 para días laborales, y en la Gráfica 2 para días festivos.

Figura 1.

Carga final promedio días laborales.

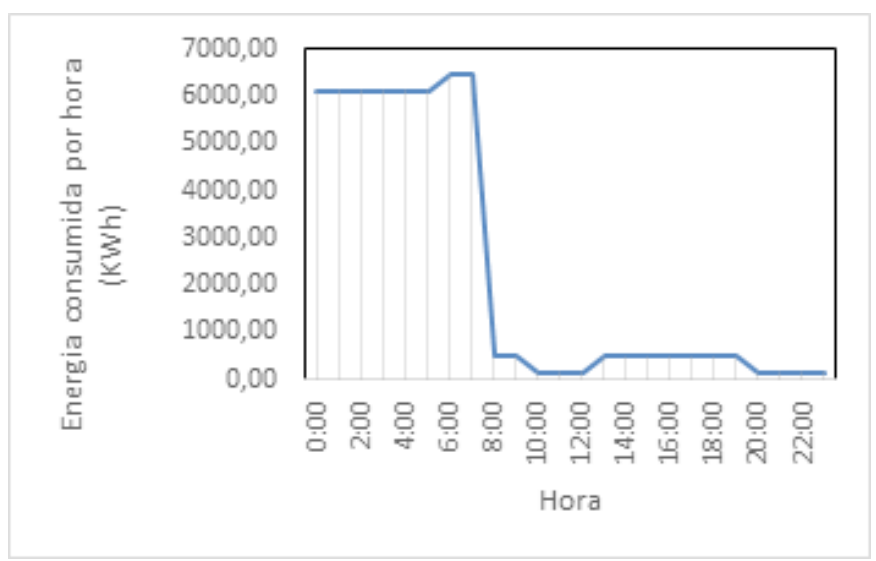

Como se puede observar en la Gráfica ,1 la sección tiene un consumo prácticamente durante todo el día, teniendo un pico entre las 0:00 y las 08:00. Por otro lado, en los días festivos (Gráfica 2) se observa un pico mayor entre las 14:00 y las 18:00, lo cual indica que en estos días el consumo es más prolongado. 
En la Gráfica 3 se muestra la desagregación de las curvas de los distintos procesos significativos que conforman la parte auditada de la planta sin contar la molienda, ya que este último requiere la mayor demanda energética de la sección. De esta forma, es más sencillo apreciar el comportamiento de los distintos procesos a lo largo del día. Los gráficos se corresponden con los días laborables.

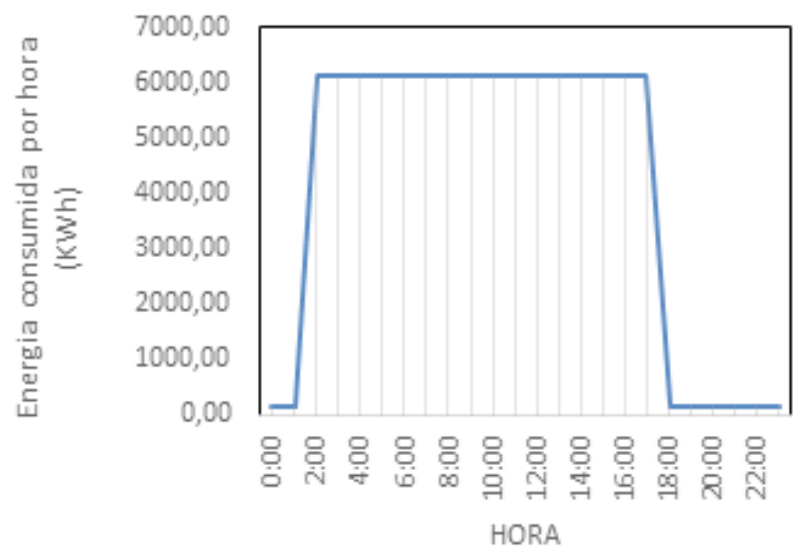

Figura 2.

Carga final promedio días festivos.

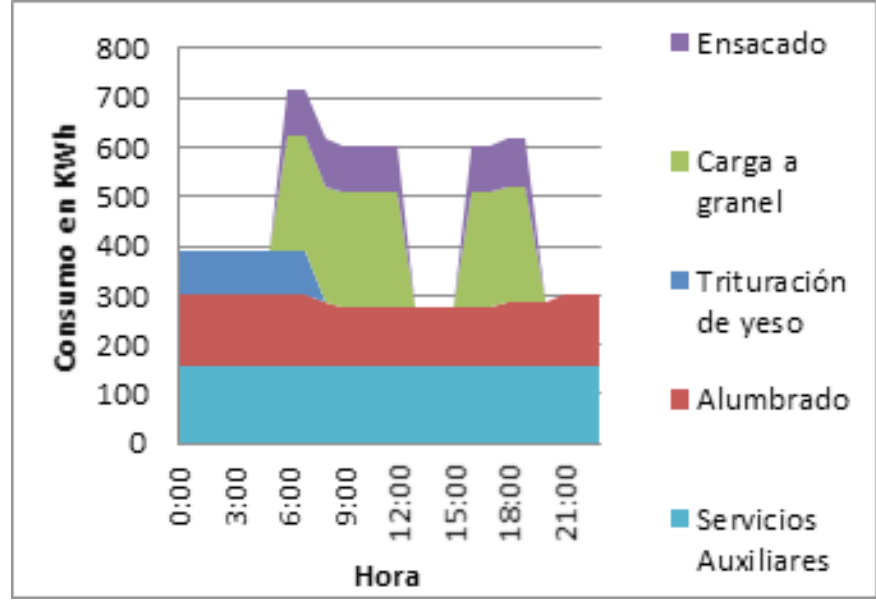

Vol 5 No2 / Julio a Diciembre 2017 Propuesta de mejora energética para una planta de producción de clinker
Figura 3.

Curva de carga por prcesos. 
Los consumos globales se distribuyen como se muestra en la Gráfica 4, donde la porción más grande corresponde a la molienda. Se evidencia así que es el proceso que más energía demanda. Uno de los grandes inconvenientes es la complejidad para modificar el proceso de molienda, por lo cual este proceso se deja a un lado y se analizan los otros 5, que son fácilmente modificables.

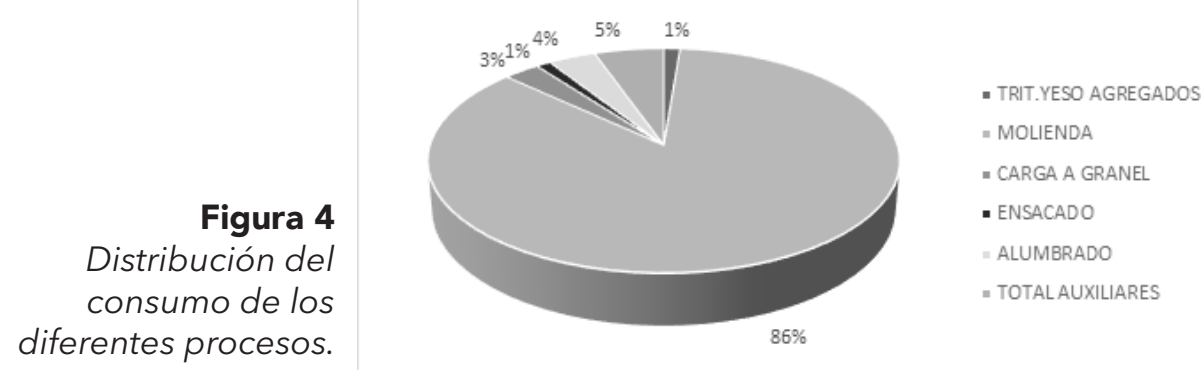

Figura 5.

Prosesos sin molienda.

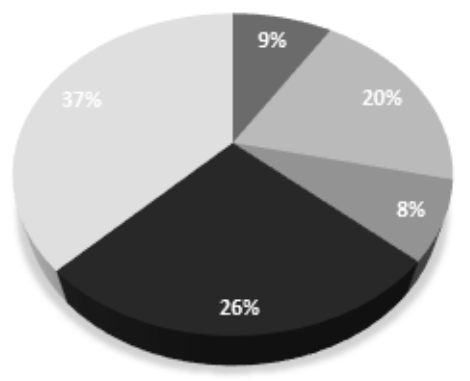

- TRIT.YESO AGREGADOS

= CARGA A GRANEL

ENSACADO

- ALUMBRADO

TOTALAUXILIARES

En la Gráfica 5, por su parte, se observa la misma distribución, y en ella se omite de nuevo la molienda. En esta Gráfica se observa claramente que el alumbrado y los auxiliares son los procesos más susceptibles de mejora. 


\section{Molienda}

Como ya se ha afirmado, el mayor consumo de la planta de cemento se concentra en la línea de molienda. Las medidas en esta línea son escasas y suelen alcanzar precios prohibitivos. Sin embargo, existe un separador que se puede reemplazar por uno de alta eficiencia. El separador actual es de tipo estático de aire, y utiliza un motor de $120 \mathrm{kw}$ con regulador de frecuencia incluido.

En todas las fábricas de cemento del mundo se hacen grandes esfuerzos para reducir los gastos de producción y, en consecuencia, del consumo de energía. Esto ha traído consigo el interés de moler el cemento en circuito cerrado con separadores de alta eficacia, lo cual ofrece varias ventajas en relación con la molienda en circuito cerrado con separadores convencionales o con la molienda en circuito abierto.

La ventaja principal es el ahorro de energía. Si el cemento se muele hasta cierto residuo de tamizado que, para una calidad de clinker dada, por lo general está en relación con las resistencias del cemento, el ahorro de energía que se puede obtener moliendo en circuito cerrado en vez de en circuito abierto es directamente proporcional al grado de eficacia del separador de aire para una determinada circulación.

Alternativamente, si se mantiene el consumo específico de energía, la utilización de un separador de alta eficacia da lugar a un cemento de mayor finura, o sea, con mejores propiedades de resistencia.

Entre las distintas opciones de separadores de alta eficiencia existentes en el mercado, se sugiere optar por un separador SEPAX, sistema que ha demostrado un excelente comportamiento en otras cementeras. 


\section{Exhaustores}

Otra opción a tomar en cuenta para el proceso de la molienda es instalar variadores de frecuencia en los motores de los exhaustores. En la planta de ensaque, existen unos colectores de aire llamados exhaustores instalados después del separador para filtrar las partículas pequeñas de cemento. Dicho cemento recogido es llevado nuevamente a las máquinas rotativas. El uso de los exhaustores permite reutilizar el cemento que se ha perdido durante el ensaque, el cual ha caído en la etapa de llenado. Actualmente en la planta del proceso de molienda de cemento, que es la parte auditada, existen 2 exhaustores constituidos por un cuerpo motor-ventilador. La figura siguiente muestra el uso de los exhaustores en el molino de cemento.

Estos dos exhaustores, como se comentó anteriormente, son muy necesarios en el proceso final. Sin embargo, tienen un gran inconveniente y es que los motores de los ventiladores siempre están encendidos y el proceso de regulación se realiza mediante una compuerta que siempre está al 90\% de apertura.

Lo anterior implica una única regulación mecánica que incrementa el consumo de estos motores, pues siempre trabajan a la máxima velocidad. La mejora a realizar consiste en implementar variadores de frecuencia a cada motor, a fin de controlar el flujo de aire eléctrico sobre el motor y no de manera mecánica, como se hace actualmente. 


\section{Compresores}

En la instalación de aire comprimido se utilizan dos compresores que permanecen rodando aun cuando no tengan que suministrar presión. Según el fabricante, el consumo de este tipo de compresores en vacío comprende aproximadamente el $45 \%$ de su potencia nominal en carga. En consecuencia, se propone introducir un controlador que los desconecte cuando no estén realizando trabajo útil y que gestione adecuadamente el reparto de la carga.

\section{lluminación}

En este caso, la medida se ha de llevar a cabo en la nave de ensacado, donde 224 luminarias de vapor de mercurio permanecen encendidas las 24 horas en los días laborables.

Como veremos a continuación, la medida planteada es una doble medida . Por una parte, se incorporarían detectores de presencia, de manera que se disminuyan las horas de consumo. De esta manera, siguiendo el horario laboral de los trabajadores de la nave de ensacado. La idea es que tras la instalación de los detectores solo se mantengan las luces conectadas entre las 6:00 y las 13:00 horas, y entre las 16:00 y las 20:00 horas.

Por otra parte, las luminarias actuales, de vapor de mercurio, se deben sustituir por luminarias de inducción, que presentan diversas ventajas, entre ellas que tiene mucha mayor eficiencia lumínica y que el tiempo de vida es mucho mayor. Así pues, para conseguir aproximadamente la misma luminosidad, estas lámparas de inducción consumirán 112 vatios, siendo esta su potencia unitaria. 


\section{Resultados}

Los resultados mostrados en esta sección corresponden al análisis de las mejores propuestas según el ahorro económico y los costos de implementación. Los resultados se basan en la Tasa Interna de Retorno TIR, utilizando el costo de la implementación y el costo del consumo, según sea el caso de la tecnología implementada, versus el costo de la tecnología a implementar y su ahorro económico por consumo.

Molienda : El ahorro energético se determinó restando el consumo energético sin la medida, menos el consumo con la medida. Tras esta operación se obtiene un ahorro de $1459317,24 \mathrm{kWh}$ al año, lo cual genera un beneficio anual de $€ 70864,44$ y un periodo de retorno de 1,06 años.

Exhaustores : Respecto a los exhaustores, el ahorro energético fue de $821453 \mathrm{kWh} /$ año, con lo cual se obtiene un ahorro económico de $€ 37524$ / año y una TIR de 3,77 años. Por otro lado, con ello se logra una reducción significativa de 533944,45 ton CO2 / año.

Compresores: $\mathrm{Si}$ los compresores se apagaran, el ahorro energético sería de $258060 \mathrm{kWh} /$ año con un beneficio económico de $€ 21935,8$, además de una disminución total de 17334,2 ton CO2/año.

Iluminación: Haciendo las mejoras propuestas en esta materia, el ahorro neto energético sería de $237667 \mathrm{kWh} /$ año, lo que significa un beneficio económico de $€ 18435$ y una disminución en las emisiones de $\mathrm{CO} 2$ de 157345 Ton de CO2/año. 


\section{Conclusiones}

El estudio presenta diferentes porcentajes de consumo de energía en las unidades estudiadas. Con las mejoras propuestas se observa una disminución significativa en la cantidad de energía consumida por la sección estudiada en 2776497,24 $\mathrm{kWh} / \mathrm{año}$. En suma, con las mejoras y a la disminución energética, se obtiene un beneficio en Euros de $€ 148489,24$, precisándose que el retorno de la inversión es menor a 5 años. Todo esto viabiliza el desarrollo de la propuesta. 


\section{Referencia Bibliográfica}

1. ONU, Declaración del Milenio, 2000.

2. G. Krajačić, R. Martins, A. Busuttil, N. Duić, \& M.D Carvalho, “Hydrogen as an energy vector in the islands' energy supply", International Journal of Hydrogen Energy, vol. 33, n.4, pp. 1091-1103.,2008.

3. C.J. Winter, "Hydrogen energy - Abundant, efficient, clean: A debate over the energy-system-of-change", International Journal of Hydrogen Energy, vol. 34, n. 14, pp. S1-S52., 2009.

4. P.J León, E. L. Martinez, A R. Bineli, M R.Wolf, F.R Maciel, “Engineering and Computer Science", 2010.

5. H. Knuutila, ErikT.Hessen, InnaKim, ToreHaug-Warberg, \& HallvardF. Svendsen, "Vapor-liquid equilibrium in the sodium carbonate-sodium", Chemical Engineering Science, 65, 2218-2226.

6. L. Rodríguez, J. Valencia, D. Rodríguez, A. Martinez, “Design, implementation and operation of a solar hybrid system in a remote area in the Colombian Guajira desert." WIT Transactions on Ecology and The Environment, Vol 195, pp. 427-438, 2015.

7. A. B. Jaffe, \& R. N. Stavins, "The energy-efficiency gap What does it mean?", Energy Policy, vol. 22, n. 10, pp. 804-810., 1994.

8. S. Cui, A. Goldsmith, \& A. Bahai, Energy-efficiency of MIMO and cooperative MIMO techniques in sensor networks. Selected Areas in Communications, IEEE Journal, vol. 22, nº. 6, pp. 1089-1098., 2004.

9. P. J León, E. L. Martinez, M R.Wolf., y F.R. Maciel, "Heat Transfer Study of Heat-Integrated Distillation Column (HIDiC) Using Simulation Technologies", IAENG Transaction of Engineering Technologies, Vol. 6. (1373), pp. 242 - 254., 2011.

10. N. Madlool, R. Saidur, M. Hossain, \& N. Rahimb, “A critical review on energy use and savings in the cement industries", Renewable and Sustainable Energy Reviews, vol. 15, n.4, pp. 2042-2060., 2011.

11. L.M. Guevara, L. Rodríguez, D. Gomez., y J.G. Chenet, “Green procurement model using petri nets: a perspective developed from the models applied to the supply chain", WIT Transactions on Ecology and The Environment, Vol 195, pp. 267-277, 2015 
12. L. Rodríguez, E. García, F. Morant, A. Correcher., \& E. Quiles, " Hybrid Analysis in the Latent Nestling Method Applied to Fault Diagnosis" IEEE Transactions on Automation Science and Engineering, vol.10, n.99, pp.1-16,2012. 13. E. Orozco Acosta, "Simulación en tiempo discreto de un proceso de abastecimiento de combustible como una herramienta de toma de decisiones: Caso estación de servicios en Barranquilla", Dictamen Libre, no. 1011, pp. 7-13, 2012. 


\section{Este artículo se cita}

L. Rodriguez, J. León, A. Cruz, H. Murcia, M. Gómez, D.

Rodríguez., y N. Lima, "Propuesta de Mejora Energética para una

Planta de Producción de Clinker", Investigación e Innovación en Ingenierias, vol. $5, \mathrm{n}^{\circ} .2$, pp. 10-25., 2017 